\title{
Fauna edáfica no processo de revegetação de áreas de mineração de bauxita em Porto Trombetas, Pará
}

\author{
Soil fauna at land revegetation in bauxite mining areas at Porto Trombetas, PA \\ Maria Elizabeth Fernandes Correia ${ }^{\mathrm{I}}$, Joventino Fernandes Moreira ${ }^{\mathrm{II}}$, \\ Luciano Lopes Reis ${ }^{\mathrm{III}}$, Fernanda de Lima Camilo ${ }^{\mathrm{IV}}$, \\ Khalil de Menezes Rodrigues ${ }^{\mathrm{v}}$, Eduardo Francia Carneiro Campello ${ }^{\mathrm{vI}}$, \\ Sérgio Miana de Faria ${ }^{\mathrm{vI}}$, Guilherme Montandon Chaer ${ }^{\mathrm{VII}}$, \\ Alexander Silva de Resende ${ }^{\mathrm{VI}}$
}

\begin{abstract}
Resumo
O trabalho objetivou acompanhar a colonização da comunidade da fauna edáfica, como indicador de qualidade de ambiente, após a revegetação de áreas alteradas pela atividade de mineração de bauxita, em Porto Trombetas, Pará. As amostragens foram realizadas nos anos 2000, 2002, 2003, 2004 e 2008 em plantios de diferentes idades, e com diferentes arranjos de espécies, majoritariamente nativas do bioma, implantadas sobre subsolo com e sem adição de solo superficial. Os organismos da fauna edáfica foram capturados em armadilhas do tipo pitfall e triados no laboratório. Os parâmetros usados para avaliação foram a abundância, a riqueza, os índices de diversidade de Shannon e Equabilidade de Pielou e o padrão de colonização dos indivíduos da fauna do solo. De modo geral, em todos os plantios sobre o estéril, os grupos que apareceram em maior abundância foram Collembola, Formicidae e Coleoptera. A idade do plantio e a adição de solo superficial foram fatores determinantes na recomposição da comunidade edáfica, que se mostrou mais intensa nos plantios mais antigos. Nessas áreas, o desenvolvimento da vegetação levou à maior colonização e à melhor estruturação da comunidade de fauna edáfica, aumentando sua diversidade e o restabelecimento de processos ecológicos.
\end{abstract}

Palavras-chave: Leguminosas; Recuperação de áreas degradadas; Indicadores biológicos

\begin{abstract}
This research aims to observe the colonization process of edaphic fauna community after the revegetation of degraded areas by bauxite miningin Porto Trombetas, Pará state, Brazil. The soil fauna samples were collected in the years of 2000,2002, 2003, 2004 and 2008, in different reforestation stages, and planted on subsoil with and without the addition of topsoil. The samplings were collected in pitfall traps and analyzed in laboratory. The evaluation parameters used were abundance, richness, the indexes of Shannon diversity and Pielou equability and the colonization pattern of individuals from the soil fauna. The planting age and the topsoil addition were determining factors in the restoration of the edaphic community, which was more intense in older plantings. In areas that went through older reforestation procedures, vegetation development has led to greater colonization and better structuring of the edaphic fauna community, increasing their diversity and restoring ecological processes.
\end{abstract}

Keywords: Leguminous plants; Reclamation of degraded areas; Biological indicators

Bióloga, Dra., Pesquisadora da Embrapa, Centro Nacional de Pesquisa de Agrobiologia, BR 465, Km 7, CEP 23890-000, Seropédica (RJ), Brasil. elizabeth.correia@embrapa.br (ORCID: 0000-0003-1919-6659)

Engenheiro Agrônomo, Dr., Professor do Instituto Federal de Educação, Ciência e Tecnologia de Alagoas, CEP 57820-000, Murici (AL), Brasil. joventinofm@gmail.com (ORCID: 0000-0001-8577-1389)

III Engenheiro Florestal, Dr., Analista Ambiental do Instituto Chico Mendes de Conservação da Biodiversidade, ICMBio, EQSW 103/104, Bloco "C", Complexo Administrativo, Setor Sudoeste CEP 70670-350, Brasília (DF), Brasil. luciano.reis@icmbio.gov.br (ORCID: 0000-0001-5076-3872)

IV Bióloga, Ma., Professora do Instituto Federal de Educação, Ciência e Tecnologia do Piauí, Campus Corrente, Rua Projetada Seis, 380, CEP 64980000, Nova Corrente (PI), Brasil. fernanda.camilo@ifpi.edu.br (ORCID: 0000-0001-7725-7689)

Engenheiro Agrônomo, Dr., Professor do Centro de Ciências Agrárias e Ambientais da Universidade Federal do Maranhão, BR 222, KM 04, s/n, Boa Vista, CEP 65500-000, Chapadinha (MA), Brasil. agrokhalil@yahoo.com.br (ORCID: 0000-0002-2650-1570)

v1 Engenheiro Florestal, Dr., Pesquisador da Embrapa, Centro Nacional de Pesquisa de Agrobiologia, BR 465, Km 7, CEP 23890-000, Seropédica (RJ), Brasil. eduardo.campello@embrapa.br (ORCID: 0000-0003-4484-9738) / sergio.defaria@embrapa.br (ORCID: 0000-0001-5066-046X) / alexander. resende@embrapa.br (ORCID: 0000-0002-1433-9203)

VII Engenheiro Agrônomo, Dr., Pesquisador da Embrapa, Centro Nacional de Pesquisa de Agrobiologia, BR 465, Km 7, CEP 23890-000, Seropédica (RJ), Brasil. guilherme.chaer@embrapa.br (ORCID: 0000-0003-0734-2866) 


\section{Introdução}

As atividades de extração mineral ocasionam mudanças significativas no ambiente, principalmente por removerem, em profundidade, uma grande quantidade de terra. A mineração de bauxita, precursora da alumina e do alumínio, se enquadra no caso descrito. O Brasil é o terceiro maior detentor das reservas de bauxita no mundo, com boa parte delas concentrada no estado do Pará, e o terceiro maior produtor com cerca de $13 \%$ da produção mundial (SUMÁRIO MINERAL, 2016).

O processo de lavra de bauxita a céu aberto consiste na remoção da vegetação, empilhamento do solo superficial, retirada da camada de substrato até cerca de $8 \mathrm{~m}$ de profundidade e finalmente a retirada da bauxita de 8 a13 m abaixo da superfície do solo. Após a extração, o substrato inicialmente retirado é retornado às cavas. Essas áreas são denominadas de estéril e precisam ser revegetadas para cumprir exigências do licenciamento ambiental das atividades e amenizar as condições de exposição do substrato (LAPA, 2000).

Diversas são as estratégias possíveis de revegetação dessas áreas. Entre elas, destaca-se o plantio de leguminosas inoculadas com bactérias fixadoras de nitrogênio e fungos micorrízicos arbusculares, precedido ou não da adição de solo superficial. O uso dessa família botânica destaca-se por ter espécies que se adaptam às mais diferentes condições de solo e clima e, quando associadas a esses microrganismos tendem a apresentar rápida cobertura do solo, favorecendo a estruturação do ambiente, para a chegada de espécies mais exigentes em umidade e fertilidade do solo e sombreamento (CHAER et al., 2011).

Além do estabelecimento da vegetação nesses ambientes, faz-se necessário o uso de ferramentas de monitoramento para avaliação dos processos ecológicos, visando avaliar o sucesso da intervenção. Os bioindicadores, por serem sensíveis aos diferentes impactos ambientais, além de bem correlacionados com as funções do solo, tornam-se fundamentais nesses casos (DORAN;ZEISS, 2000). O monitoramento da fauna do solo em áreas em recuperação poderá fornecer informações do funcionamento dos novos ecossistemas criados a partir da intervenção.

Poucos estudos têm sido feitos para avaliar indicadores de qualidade do solo em áreas de mineração, a longo prazo. Essa oportunidade foi vislumbrada neste trabalho que objetivou avaliar a colonização da comunidade de fauna edáfica em plantios de diferentes idades, sobre estéril de bauxita, com e sem aplicação de solo superficial e revegetados com espécies florestais com forte presença de leguminosas arbóreas e arbustivas associadas a microrganismos.

\section{Material e método}

\section{Caracterização das áreas, do processo de revegetação e dos tratamentos}

O trabalho foi conduzido em áreas localizadas no platô Saracá, no interior da Floresta Nacional de Saracá-Taquera, localizada a cerca de $30 \mathrm{~km}$ do distrito de Porto de Trombetas, no município de Oriximiná - PA, sob as coordenadas $1^{\circ} 40^{\prime} 30^{\prime \prime}$ S e $56^{\circ} 27^{\prime} 13^{\prime \prime}$ W. De acordo com a classificação de Köppen, o clima é do tipo AW, com estação seca e chuvosa bem definidas, com precipitação pluviométrica anual variando de 2.500 a $3.000 \mathrm{~mm}$ (FERRAZ, 1993).

Nas áreas avaliadas, após a disposição do estéril da mineração de bauxita por toda a área a ser revegetada, foi realizado o nivelamento e reposição do solo superficial proveniente da abertura de novas alocações. O plantio foi efetuado com uma mistura de espécies nativas, sendo muitas leguminosas fixadoras de nitrogênio, no espaçamento de $2 \mathrm{~m} \times 2 \mathrm{~m}$. A adubação foi preparada na cova, com $150 \mathrm{~g}$ de calcário dolomítico, $130 \mathrm{~g}$ de adubo formulado NPK (4-14-8), $40 \mathrm{~g}$ de termofosfato e $10 \mathrm{~g}$ de micronutriente FTE BR-12. O plantio foi realizado em linhas, em covas de $0,2 \mathrm{~m} \times 0,2 \mathrm{~m}$. A inoculação das leguminosas com bactérias específicas procedeu-se na fase de produção das mudas, a partir da aplicação de inoculante turfoso nas sementes, fornecido pela Embrapa Agrobiologia.

Neste trabalho foram considerados os plantios realizados nos anos de 1984, 1992, 1994, 1999, todos com adição de solo superficial e, uma única área plantada em 1994, na qual este não foi aplicado. As áreas são próximas entre si, estando todas em um raio de 7 x $7 \mathrm{~km}$. O tamanho das áreas era variável, 
mas sempre superior a 2 hectares. O número de espécies plantadas em cada ano foram 67, 91, 97 e 146, respectivamente. A listagem com as espécies está descrita em Salomão et al. (2007) e Moreira (2010). Para fins de notação, os plantios foram identificados com ano em que foram plantados e no caso do plantio sem solo superficial, para diferenciar, foi nomeado de 1994 SS (sem adição de solo superficial).Para fins de comparação com as áreas reflorestadas foram avaliadas amostras retiradas de um remanescente de floresta nativa, localizado próximo à área de mineração, no platô Saracá, identificada como Mata.

As amostragens foram realizadas sempre no segundo semestre de cada ano, em novembro de 2000 , setembro de 2002, dezembro de 2003, agosto de 2004 e novembro de 2008 , coincidindo com a estação seca que vai de julho a dezembro. Os dados de precipitação do período encontram-se na Figura 1. Para a coleta da fauna do solo epígea, em cada sistema avaliado, foram instaladas armadilhas do tipo pitfall, dispostas ao longo de um transecto com intervalos de $5 \mathrm{~m}$, para a captura dos organismos (MOLDENKE, 1994). Utilizaram-se sete repetições por área, totalizando 42 amostras, incluindo a área de Mata. Esse modelo de armadilha possui $9 \mathrm{~cm}$ de diâmetro por $11 \mathrm{~cm}$ de altura e foi inserida com a borda ao nível do solo. Como líquido coletor foi utilizado solução aquosa de formol a $4 \%$. As armadilhas permaneceram no campo durante sete dias, quando foram recolhidas e o seu conteúdo transferido para frascos contendo álcool $70 \%$. No laboratório, as amostras foram triadas sob lupa binocular e a fauna identificada em ordens ou famílias, a partir de chaves de classificação existentes no Laboratório de Fauna do Solo, da Embrapa Agrobiologia. Com os resultados determinou-se a abundância (número de indivíduos armadilha ${ }^{-1} \mathrm{dia}^{-1}$ ).

\section{Figura 1 - Precipitação ( $\mathrm{mm}$ de chuva) no período de quatro meses que precederam as coletas de fauna epígea, nos anos de 2000 a 2008, Porto Trombetas, PA}

Figure 1 - Precipitation ( $\mathrm{mm}$ of rainfall) in the period that preceded collections of epigeal fauna, from 2000 to 2008 , Porto Trombetas, PA

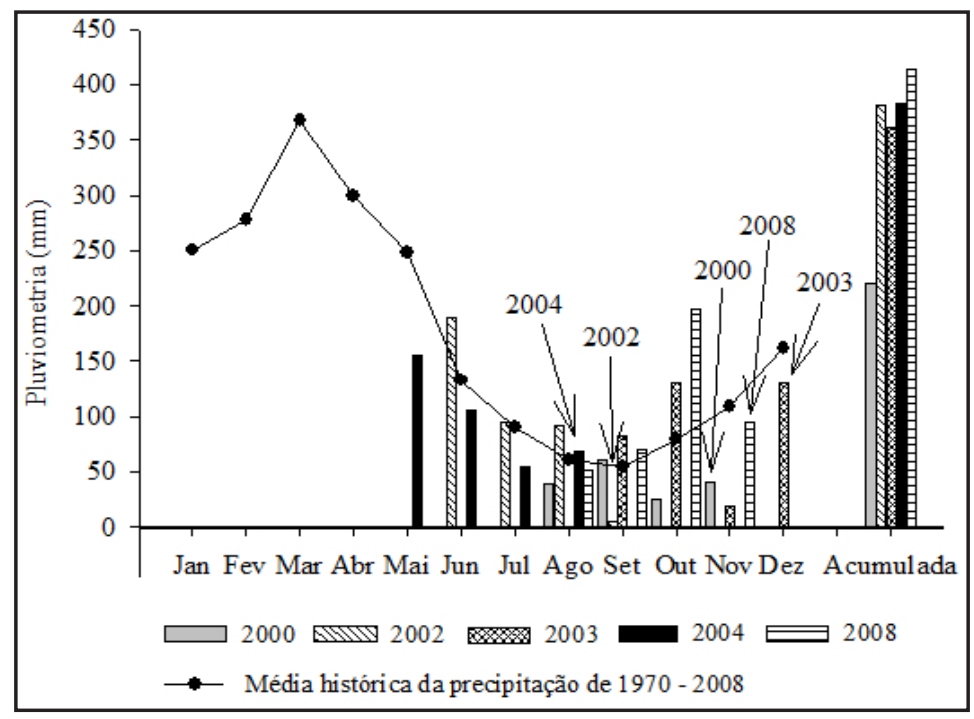

Fonte: Mineração Rio do Norte (2008)

\section{Análises estatísticas}

Para determinação da atividade da fauna do solo, o número de indivíduos em cada coleta foi dividido pelo número de armadilhas (7) e de dias no campo (7) para obter a atividade dos indivíduos por armadilha dia $^{-1} \mathrm{e}$ foi estimado também o seu respectivo erro padrão. Calcularam-se os índices ecológicos de Shannon e Pielou, além da riqueza total e média. A abundância e a riqueza médias foram comparadas em cada tratamento através do teste $t$ de Bonferroni.

Para obtenção do perfil da fauna do solo, os dados foram submetidos à análise multivariada com base na técnica de Escalonamento Multidimensional Não Métrico (NMS) (KRUSKAL, 1964a e b), para 
avaliar o padrão de evolução da comunidade de fauna edáfica ao longo dos anos. Antes da ordenação, os dados foram relativizados pelo total de indivíduos coletados em cada armadilha, expressando a composição relativa dos grupos de fauna do solo em percentagem.

A área de Mata foi ordenada juntamente com as demais e foi usada como área de referência. Todas as ordenações foram rotacionadas de forma a posicionar a Mata à direita do gráfico, fazendo com que o grau de similaridade do perfil da fauna do solo obtido nas áreas em recuperação fosse expresso principalmente no eixo 1 da ordenação. Dessa forma, considerou-se que os plantios com a comunidade edáfica menos desenvolvida se posicionam à esquerda do gráfico, enquanto os plantios com melhor estabelecimento da fauna do solo posicionam-se à direita, formando, assim, um gradiente de similaridade em relação à Mata.

As diferenças estatísticas na composição da fauna entre as áreas foram avaliadas por procedimentos de permutação multiresposta (MRPP) (BIONDINI et al., 1985). As relações entre os perfis da fauna do solo obtidos na ordenação e a riqueza, os índices de Shannon e de Pielou foram avaliados por meio da correlação de Pearson entre essas características e os eixos da ordenação NMS.

Ainda foi utilizada a Análise de Espécies Indicadoras (VI) conforme Dufrênee Legendre (1997), como ferramenta para determinação do "valor de bioindicação". Essa técnica aponta preferências das espécies por habitat que apresente características ambientais específicas. O método combina informação sobre a concentração da abundância de uma espécie em um determinado grupo de unidades amostrais e da fidelidade da ocorrência dessa espécie em certo grupo de amostras, ou seja, é uma combinação de abundância relativa com frequência relativa através do grau de especificidade de uma determinada espécie para um status ecológico, por exemplo, tipo de habitat e sua fidelidade dentro do status, medida através da sua percentagem de ocorrência (MCGEOCH et al., 1998).

Finalizando, empregou-se o índice $\mathrm{V}$ ou Índice de Mudança, com o objetivo de investigar possíveis alterações na abundância/atividade da comunidade da fauna do solo como resultado da influência dos tratamentos testados (WARDLE, 1995). Este índice foi calculado pela Equação (1):

$$
V=\left(\frac{2 x}{x}+y\right)-1
$$

onde: $\mathrm{V}$ = índice $\mathrm{V} ; \mathrm{x}=$ abundância dos grupos em cada tratamento; $\mathrm{y}=$ abundância dos grupos na área de referência (Mata). O índice $\mathrm{V}$ varia de -1 a 1 e, quanto mais próximo o valor for de 1, maior foi a estimulação da abundância dos grupos da fauna do solo, no tratamento aplicado. Em contrapartida, quanto mais próximo o valor for de -1, maior foi a inibição da abundância. Valores compreendidos entre - $-0,05$ e 0,05 indicam que a abundância não foi alterada pelo tratamento aplicado, em comparação com a situação original ou ideal. De acordo com os valores encontrados, o efeito dos tratamentos sobre a abundância dos grupos foi classificado em categorias (Tabela 1).

\section{Tabela 1 - Categorias de inibição e estimulação dos grupos da fauna de solo em resposta ao processo de intervenção, com base no índice $\mathrm{V}$}

Table 1 - The soil fauna groups inhibition and stimulation categories in response to the intervention process, based on the $\mathrm{V}$ index

\begin{tabular}{lcc}
\hline \multicolumn{1}{c}{ Categoria } & Símbolo & Índice V \\
\hline Inibição extrema & IE & $\mathrm{V}<-0,67$ \\
Inibição moderada & IM & $-0,33>\mathrm{V}<-0,67$ \\
Inibição leve & IL & $-0,05>\mathrm{V}<-0,33$ \\
Sem alteração & $\mathrm{SA}$ & $-0,05>\mathrm{V}<0,05$ \\
Estimulação leve & $\mathrm{EL}$ & $0,05>\mathrm{V}<0,33$ \\
Estimulação moderada & $\mathrm{EM}$ & $0,33>\mathrm{V}<0,67$ \\
Estimulação extrema & $\mathrm{EE}$ & $\mathrm{V}>0,67$ \\
\hline
\end{tabular}

Fonte: Autores (2010), modificado de Wardle (1995) 


\section{Resultados e discussão}

O ordenamento multivariado dos dados de fauna do solo resultou em dois eixos que explicaram juntos $86 \%$ da variância total. Houve diferenças no padrão de colonização dos organismos em cada plantio ao longo dos anos de amostragem, sendo que os mais antigos 1984, 1992 e 1994 apresentaram maior estabilidade em relação aos plantios de 1994 SS e de 1999 (Figura 2). O perfil da fauna do solo dos plantios de 1992 e de 1994 apresentava-se em um patamar mais elevado desde a primeira amostragemem 2000. Isso se deu em função de grande parte da fauna do solo já estar estabelecida, considerando o tempo maior de desenvolvimento da vegetação. Por outro lado, o plantio de 1999 tinha apenas 1 ano de idade por ocasião da primeira amostragem, o que levou a expressão de dados com muita variabilidade, mostrando instabilidade do indicador fauna do solo em função do curto período de estabelecimento da vegetação em relação às demais áreas.

Figura 2 - Mudanças no perfil da fauna do solo entre os anos de 2000 e 2008 em plantios sobre estéril de mineração de bauxita. Os perfis foram representados pelo eixo 1 do ordenamento multidimensional não métrico (NMS), correspondente às diferenças em relação à mata nativa em cada ponto. As barras verticais correspondem ao erro padrão. Os asteriscos indicam que os valores encontrados na Mata, não diferiram dos valores obtidos nos plantios assinalados naquele ano

Figure 2 - Changes in the soil fauna profile between the years of 2000 and 2008 at plantations on barren bauxite mining subsoil. The profiles were represented by axis 1 of the multidimensional non-metric order (NMS), corresponding the differences in relation to the native forest at each point. The vertical bars correspond to the standard error and the asterisks indicate that there was no statistical difference between the planting and the forest in each sampling season

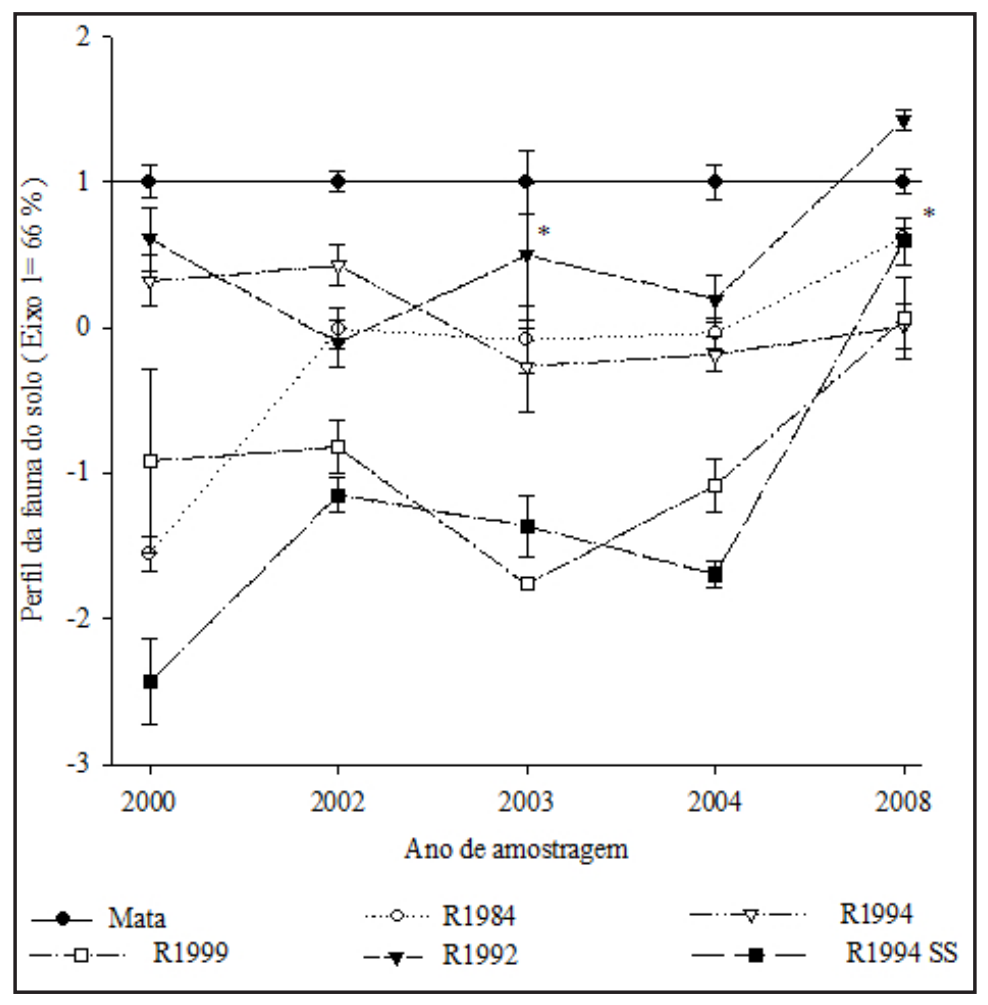

Fonte: Autores (2010) 
Através do ordenamento por NMS, no que se refere aos índices avaliados, a riqueza de grupos foi a que apresentou maior correlação com o ordenamento das áreas $(r=0,71)(p<0,001)$ (Tabela 2), indicando a ocorrência de progressão dos dados que expressam a riqueza no sentido de aproximar os plantios da mata nativa com o tempo de desenvolvimento dos mesmos. Assim, nos plantios de 1994 SS e 1999, foi observado aumento nos valores de riqueza da primeira para a última avaliação (Figura 2), o que demonstra o estabelecimento de novos grupos da fauna do solo à medida que os plantios se desenvolveram.

\section{Tabela 2 - Coeficientes de correlação de Pearson $(\mathbf{r})(\mathbf{n}=189)$ dos índices avaliados em relação aos escores do eixo 1 do NMS}

Table 2 - Pearson correlation coefficients $(r)(n=189)$ of the evaluated indicesin relation to the NMS axis 1 scores

\begin{tabular}{|c|c|}
\hline Índices & Eixo 1 \\
\hline Abundância & $0,55^{\text {thk }}$ \\
\hline Riqueza total (RT) & $0,71^{\text {tht }}$ \\
\hline Índice de Shannon $(\mathbf{H})$ & $0,43^{*+k+}$ \\
\hline Índice de Pielou (U) & $0,22^{* *}$ \\
\hline
\end{tabular}

Fonte: Autores (2010)

Em que: Valores seguidos por ${ }^{* *} \mathrm{e}^{* \star *}$ indicam significância estatística ao nível de $\mathrm{p}<0,01$ ep $<0,001$, respectivamente, pelo teste t de Bonferroni.

A riqueza total (RT), que representa o número total de grupos taxonômicos encontrados na área, se mostrou como bom parâmetro de avaliação por ter apresentado maior correlação com o eixo 1 e por ser mais discriminante em relação aos tratamentos. Nesse sentido, enquanto a Mata sempre apresentou valores próximos ou superiores a 20 grupos em todas as amostragens, nos plantios, valores nessa faixa só ocorreram na avaliação realizada no ano de 2008 (Tabela 3). Nos plantios de 1994 SS e no de 1999 foram verificados sempre valores abaixo de 10 até o ano de 2008. Por outro lado, os plantios 1984, 1992 e 1994 sempre se destacaram em relação aos demais, com riqueza superior a 15 grupos, em média. O maior tempo de plantio e a adição de solo superficial, possivelmente explicam esses resultados.

A abundância e os índices de Shannon e Pielou também apresentaram correlações positivas com o eixo 1 , com valores de 0,$55 ; 0,43$ e 0,22 , respectivamente (Tabela 2). No caso da abundância, foi possível notar que ocorreu grande variabilidade entre os anos de coleta (Tabela 3). A Mata apresentou abundâncias elevadas na maioria dos anos avaliados e os plantios mais antigos (1984, 1992, 1994), apesar das variações, se encontraram em um mesmo patamar de abundância e na maioria das amostragens, próximos à área de Mata, com destaque para o plantio realizado em 1992.

No caso do reflorestamento de 1994 SS, a abundância foi inferior a dos outros plantios e semelhante à encontrada no reflorestamento realizado em 1999, reforçando que a adição de solo superficial se constitui numa importante estratégia para o avanço dos processos ecológicos da fauna edáfica na área de estudo.

À medida que os plantios se desenvolveram, estes atingiram um patamar de diversidade relativamente similar ao da Mata em nível de grandes grupos da fauna do solo. É possível que em níveis taxonômicos menores, como no caso de gênero e espécie, poderia ser encontrada maior diferença entre os plantios e o ecossistema original, mas isso não foi avaliado neste estudo.

No trabalho de Salomão et al. (2007), os autores avaliaram a regeneração natural da vegetação nos mesmos tratamentos aqui apresentados. Constataram que o plantio de 1992 
foi o que apresentou o maior diâmetro médio das plantas, maior número de espécies e maior incremento da área basal. Indicando que os melhores resultados nos parâmetros fitossociológicos refletiram na fauna do solo, onde um vigoroso restabelecimento da vegetação ajuda a estruturar toda a cadeia ecológica, reforçando o conceito de espécies estruturantes (SALOMÃO, 2015).

Tabela 3 - Abundância (n. ${ }^{\circ}$ de indivíduos $\operatorname{armadilha}^{-1}$ dia $^{-1} \pm$ erro padrão) e índices ecológicos dos principais grupos da fauna do solo nos plantios, nos cinco anos amostrados, em Porto Trombetas-PA

Table 3 - Abundance (No. of individuals trap- 1 day- $1 \pm$ standard error) and ecological indexes of the soil fauna main groups at reforestations, during the five years sampled, at Porto Trombetas-PA

\begin{tabular}{|c|c|c|c|c|c|}
\hline Plantio & Abundância & Riqueza Total & Riqueza média & Índice de Shannon & Índice de Pielou \\
\hline \multicolumn{6}{|c|}{2000} \\
\hline 1984 & $22,6 \pm 4,5 \mathrm{ab}^{*}$ & 16 & $9 \mathrm{~b}$ & 1,80 & 0,44 \\
\hline 1992 & $30,3 \pm 4,2 \mathrm{a}$ & 17 & $12 \mathrm{a}$ & 2,30 & 0,56 \\
\hline 1994 SS & $18,4 \pm 3,8 \mathrm{ab}$ & 14 & $9 \mathrm{~b}$ & 2,40 & 0,62 \\
\hline 1994 & $3,8 \pm 2,7 \mathrm{~b}$ & 9 & $3 c$ & 1,40 & 0,45 \\
\hline 1999 & $17,3 \pm 7,3 \mathrm{ab}$ & 7 & $4 \mathrm{c}$ & 1,40 & 0,50 \\
\hline Mata & $31,5 \pm 4,0 \mathrm{a}$ & 18 & $12 \mathrm{a}$ & 2,60 & 0,63 \\
\hline CV (\%) & 54 & & 19 & & \\
\hline \multicolumn{6}{|c|}{2002} \\
\hline 1984 & $21,5 \pm 4,2 \mathrm{bc}$ & 16 & $9 \mathrm{a}$ & 2,20 & 0,54 \\
\hline 1992 & $16,7 \pm 2,9 \mathrm{bc}$ & 14 & $9 \mathrm{a}$ & 2,50 & 0,65 \\
\hline 1994 SS & $32,9 \pm 10,5 b$ & 17 & $11 \mathrm{a}$ & 2,40 & 0,59 \\
\hline 1994 & $5,3 \pm 1,1 \mathrm{c}$ & 6 & $2 \mathrm{~b}$ & 0,60 & 0,23 \\
\hline 1999 & $20,3 \pm 9,0 \mathrm{bc}$ & 8 & $4 \mathrm{~b}$ & 0,70 & 0,22 \\
\hline Mata & $66,5 \pm 10,5 \mathrm{a}$ & 24 & $13 \mathrm{a}$ & 1,60 & 0,34 \\
\hline $\mathrm{CV}(\%)$ & 64 & & 22 & & \\
\hline \multicolumn{6}{|c|}{2003} \\
\hline 1984 & $7,8 \pm 1,2 \mathrm{~ns}$ & 17 & $8 \mathrm{ab}$ & 2,40 & 0,60 \\
\hline 1992 & $11,5 \pm 1,1 \mathrm{~ns}$ & 20 & $11 \mathrm{a}$ & 2,40 & 0,56 \\
\hline 1994 SS & $10,3 \pm 1,9 \mathrm{~ns}$ & 16 & $8 \mathrm{ab}$ & 2,30 & 0,57 \\
\hline 1994 & $9,3 \pm 1,4 \mathrm{~ns}$ & 17 & $9 \mathrm{ab}$ & 2,00 & 0,50 \\
\hline 1999 & $5,5 \pm 0,9 \mathrm{~ns}$ & 8 & $5 \mathrm{~b}$ & 1,30 & 0,42 \\
\hline Mata & $10,6 \pm 2,1 \mathrm{~ns}$ & 21 & $11 \mathrm{a}$ & 2,90 & 0,65 \\
\hline $\mathrm{CV}(\%)$ & 40 & & 32 & & \\
\hline
\end{tabular}


Tabela 3 - Conclusão ...

Table 3 - Conclusion ...

\begin{tabular}{|c|c|c|c|c|c|}
\hline Plantio & Abundância & Riqueza Total & Riqueza média & Índice de Shannon & Índice de Pielou \\
\hline \multicolumn{6}{|c|}{2004} \\
\hline 1984 & $7,4 \pm 1,5 b$ & 14 & $8 \mathrm{~b}$ & 2,80 & 0,73 \\
\hline 1992 & $9,2 \pm 2,15 b$ & 16 & $9 \mathrm{~b}$ & 2,80 & 0,69 \\
\hline 1994 SS & $7,6 \pm 1,2 b$ & 12 & $9 \mathrm{~b}$ & 2,70 & 0,75 \\
\hline 1994 & $6,9 \pm 1,7 \mathrm{~b}$ & 9 & $4 \mathrm{c}$ & 1,30 & 0,41 \\
\hline 1999 & $7,5 \pm 1,5 b$ & 9 & $5 \mathrm{c}$ & 1,60 & 0,52 \\
\hline Mata & $15,5 \pm 2,5 \mathrm{a}$ & 21 & $11 \mathrm{a}$ & 2,60 & 0,59 \\
\hline $\mathrm{CV}(\%)$ & 92 & & 31 & & \\
\hline \multicolumn{6}{|c|}{2008} \\
\hline 1984 & $40,0 \pm 5,7 \mathrm{bc}$ & 23 & $13 \mathrm{~ns}$ & 2,50 & 0,55 \\
\hline 1992 & $69,8 \pm 6,7 \mathrm{a}$ & 23 & $13 \mathrm{~ns}$ & 1,30 & 0,28 \\
\hline 1994 SS & $59,5 \pm 9,3 \mathrm{ab}$ & 20 & $12 \mathrm{~ns}$ & 2,00 & 0,44 \\
\hline 1994 & $28,0 \pm 5,3 \mathrm{c}$ & 23 & $12 \mathrm{~ns}$ & 2,40 & 0,54 \\
\hline 1999 & $20,7 \pm 4,1 \mathrm{c}$ & 18 & $11 \mathrm{~ns}$ & 3,10 & 0,71 \\
\hline Mata & $31,5 \pm 4,0 \mathrm{c}$ & 21 & $13 \mathrm{~ns}$ & 2,70 & 0,59 \\
\hline $\mathrm{CV}(\%)$ & 39 & & 23 & & \\
\hline
\end{tabular}

Fonte: Autores (2010)

Em que: *Médias seguidas pela mesma letra, na coluna, não diferem entre si pelo teste t de Bonferroni $(p<0,05)$.

Outra questão relevante diz respeito aos plantios realizados em 1994. Na área sem o uso de solo superficial, a comunidade da fauna edáfica demorou mais tempo para se estabelecer. Além disso, apresentou a maior variabilidade a cada ano. Por outro lado, onde foi adicionado solo superficial, já na avaliação feita em 2000 , pode-se considerar que a comunidade da fauna edáfica já estava estabelecida. Nessa situação, como a degradação inicial foi muito intensa, ocorrendo a retirada de toda a vegetação, solo e subsolo, a colonização natural pode ser lenta, sendo assim o processo de estabelecimento da fauna edáfica é acelerado quando do retorno do solo superficial, trazendo propágulos e mais fertilidade ao substrato (MARTINS et al., 2019).

Em relação aos coeficientes de correlação de Pearson dos grupos de fauna do solo e a análise de espécies indicadoras (Tabela 4), observou-se que apenas Chilopoda, Homoptera, Lepidoptera e Psocoptera não apresentaram correlação significativa com o eixo 1.Os grupos que mais influenciaram a disposição das áreas no eixo do gráfico foram Collembola $(0,58)$, Coleoptera $(0,41)$, Araneae $(0,32)$, Thysanoptera $(0,32)$, Formicidae $(-0,32)$, Hymenoptera $(0,30)$ e Pseudoscorpionida $(0,26)$. Para todos esses grupos, a correlação foi altamente significativa $(\mathrm{p}<0,001)$, indicando que houve um gradiente caracterizado pelo aumento do valor de abundância dos grupos de fauna do solo dosplantios de 1999 e 1994 SS para os plantios de 1984, 1994 e 1992 e, por fim, da mata nativa. Todos esses grupos foram mais abundantes na mata nativa, com exceção de Formicidae que apresentou maior atividade nos plantios de 1999 e de 1994 SS, justamente os menos desenvolvidos. 
Tabela 4 - Coeficientes de correlação de Pearson $(\mathbf{r})(\mathbf{n}=189)$ das variáveis com os eixos 1 , obtidos a partir do ordenamento por NMS e percentual de indicação com os grupos de fauna do solo amostrados em plantios de diferentes idades plantados sobre estéril de mineração de bauxita e mata nativa, Porto Trombetas, PA

Table 4 - Pearson correlation coefficients $(r)(n=189)$ of the variables with axes 1 , obtained from NMS and indication percentage of the soil fauna groups under at different ages planted reforestations on bauxite mining subsoil and native forest, Porto Trombetas, PA

\begin{tabular}{|c|c|c|c|c|c|c|}
\hline \multirow{3}{*}{ Grupos } & \multirow{3}{*}{$\begin{array}{c}\text { Correlações NMS } \\
\text { Eixo } 1\end{array}$} & \multicolumn{5}{|c|}{ Análise de grupos indicadores } \\
\hline & & Ar & Fr & VI & \multirow{2}{*}{$p$} & \multirow{2}{*}{ Área indicada } \\
\hline & & & $\%$ & & & \\
\hline Araneae & $0,32^{* * k}$ & 22 & 97 & 21 & 0,0548 & R1992 \\
\hline Archaeognatha & $0,21^{* *}$ & 46 & 7 & 3 & 0,3595 & R1992 \\
\hline Blattodea & $0,17^{*}$ & 39 & 87 & 34 & 0,0002 & R1994 \\
\hline Chilopoda & 0,02 & 32 & 6 & 2 & 0,8704 & R1994SS \\
\hline Coleoptera & $0,41^{\text {k*k }}$ & 39 & 100 & 39 & 0,0002 & Mata \\
\hline Collembola & $0,58^{* k \hbar}$ & 25 & 100 & 25 & 0,0008 & R1992 \\
\hline Dermaptera & $0,17^{*}$ & 96 & 52 & 49 & 0,0002 & Mata \\
\hline Diplopoda & $0,16^{*}$ & 60 & 11 & 7 & 0,6509 & R1984 \\
\hline Diptera & $0,24^{\text {*k* }}$ & 27 & 97 & 27 & 0,0016 & R1984 \\
\hline Formicidae & $-0,31^{\text {*tak }}$ & 28 & 100 & 28 & 0,0002 & R1994SS \\
\hline Heteroptera & $0,19^{* *}$ & 31 & 39 & 12 & 0,2316 & Mata \\
\hline Homoptera & 0,08 & 29 & 36 & 10 & 0,6709 & R1999 \\
\hline Hymenoptera & $0,30^{* \star * *}$ & 22 & 84 & 19 & 0,1466 & R1994 \\
\hline Isopoda & $0,13^{*}$ & 51 & 20 & 10 & 0,0346 & R1984 \\
\hline Blattodea (Termitoidea) & $0,19^{* *}$ & 40 & 33 & 13 & 0,0132 & R1992 \\
\hline Larvas de Coleoptera & $0,14^{*}$ & 30 & 39 & 12 & 0,2442 & Mata \\
\hline Larvas de Diptera & $0,22^{k *}$ & 29 & 70 & 20 & 0,0204 & Mata \\
\hline Larvas de Lepidoptera & $0,19^{*}$ & 32 & 15 & 5 & 0,2428 & Mata \\
\hline Lepidoptera & 0,04 & 69 & 34 & 24 & 0,0002 & R1984 \\
\hline Opilionida & $0,18^{* *}$ & 35 & 23 & 8 & 0,0796 & R1992 \\
\hline Orthoptera & $0,17^{* *}$ & 24 & 79 & 19 & 0,2150 & R1999 \\
\hline Pseudoscorpionida & $0,26^{\text {k*kx }}$ & 81 & 27 & 22 & 0,0002 & Mata \\
\hline Psocoptera & 0,06 & 35 & 13 & 4 & 0,9200 & R1994SS \\
\hline Thysanoptera & $0,32^{\text {wat }}$ & 23 & 52 & 12 & 0,5551 & Mata \\
\hline
\end{tabular}

Fonte: Autores (2010)

Em que: " $\mathrm{p}<0,05 ;{ }^{* *} \mathrm{p}<0,01 ;{ }^{* * *} \mathrm{p}<0,001 ;$ Em que: VI=Valor indicador (\% de indicação); Ar= Abundância relativa (abundância média de uma área em relação à abundância de todas as amostras); Fr=Frequência relativa (Percentual de amostras da área em que o grupo está presente); $p=$ probabilidade do teste de indicação. 
Pela análise de possíveis grupos indicadores de qualidade de ambiente, foi verificado que, daqueles comníveis de indicação significativos pelo teste de Monte Carlo, Dermaptera e Coleoptera foram os principais, com valores de $49 \%$ e $39 \%$, respectivamente, ambos para a Mata. Em seguida destacam-se os grupos Blattodea (34\%), Formicidae (28\%) e Diptera (27\%) para 1994, 1994SS e 1984, respectivamente. Todos esses grupos foram considerados como fortes indicadores, ou seja, apresentaram valores indicadores superiores a $25 \%$ e frequência relativa superior a $50 \%$ (DUFRENE; LEGENDRE, 1997).Segundo esses autores, valores acima de $25 \%$ são simétricos, ou seja, pode-se esperar que uma espécie característica esteja presente em pelo menos $50 \%$ das amostras da área e que a sua abundância relativa naquele grupo atinja pelo menos $50 \%$, o que significa que a presença da espécie contribui para a especificidade da área e, portanto, possa ser prevista em todas as amostras da mesma. Neste estudo, grupos com VI iguais ou superiores a $25 \%$ apresentaram abundância ou constância inferior a $50 \%$ na área, sendo que apenas Dermaptera apresentou abundância relativa superior a 50\%. Nessas condições, esses grupos podem ser considerados indicadores, embora ainda com baixos níveis de confiabilidade (BAKKER, 2008).

Na Tabela 5 é apresentado um contraste entre as amostragens de 2000 e 2008 no que tange ao índice de valor de mudança da fauna do solo. Considerando o grupo taxonômico e a condição de estimulação (seja leve, moderada ou extrema), os plantios de 1984; 1992; 1994; 1994 SS e 1999 apresentaram no ano de 2000: 2, 8, 5, 3 e 2 grupos nessas classes, respectivamente. Já no ano de 2008 , os valores foram 8, 9, 7, 6 e 7, respectivamente. Isso expressa, também por esse método de avaliação, que com o passar dos anos a comunidade de fauna do solo foi mais estimulada em todos os tratamentos, indicando que o tempo é fundamental para a melhor expressão desse indicador. Um grupo a ser observado é o Pseudoscorpionida, por ser considerado de topo de cadeia trófica, e que foi estimulado nos plantios mais antigos de 1984 e 1992, somente na avaliação feita em 2008. Indicando que apesar do avanço ecológico em todos os tratamentos, só os mais antigos é que permitiram uma condição mais favorável para grupos mais exigentes.

Considerando o papel da fauna do solo na ciclagem de nutrientes, assim como na decomposição e mineralização da matéria orgânica e disponibilização dos nutrientes, de modo geral, nos plantios, é possível afirmar que os organismos do solo estiveram intimamente ligados à sua cobertura. Em florestas tropicais, um dos mais importantes mecanismos da ciclagem de nutrientes é a deposição de matéria orgânica no solo com a queda de folhas, galhos, cascas, entre outros, constituindo a base de sustentação desses ambientes, uma vez que as partes produtoras desses ecossistemas, que são as plantas, dependem principalmente dos nutrientes reciclados contidos na camada de serapilheira para sua nutrição e manutenção (GIÁCOMO et al., 2017).

Esses resultados demonstram a importância do retorno da camada de solo superficial para áreas que sofreram intervenção desse tipo, desempenhando papel fundamental no processo de revegetação, pois pode conter a "memória" do ambiente local, além de fonte de matéria orgânica e nutrientes, trazendo propágulos de plantas, microrganismos e a fauna do solo e, assim, acelerando o processo de recuperação do ambiente (LIMA et al., 2017).

O ordenamento multivariado dos dados da fauna do solo resultou em dois eixos, que juntos explicaram $86 \%$ da variância total (Figura 3). Apesar da ampla dispersão dos pontos no gráfico, é possível notar três grupos: o da mata nativa, com a maioria dos pontos localizados à direita do gráfico; o dos plantios mais antigos (1984, 1992 e 1994), mais ao centro e, mais à esquerda, os plantios de 1999 e de 1994 SS. 
Tabela 5 - Índice de valor de mudança da fauna do solo, para os diferentes anos de implantação das diferentes áreas avaliadas em estéril de bauxita, nos anos de 2000 e 2008, em Porto Trombetas, PA

Table 5 - Soil fauna change value index for the different years of reforestation implantation on bauxite mining subsoil, in the years of 2000 and 2008, at Porto Trombetas, PA

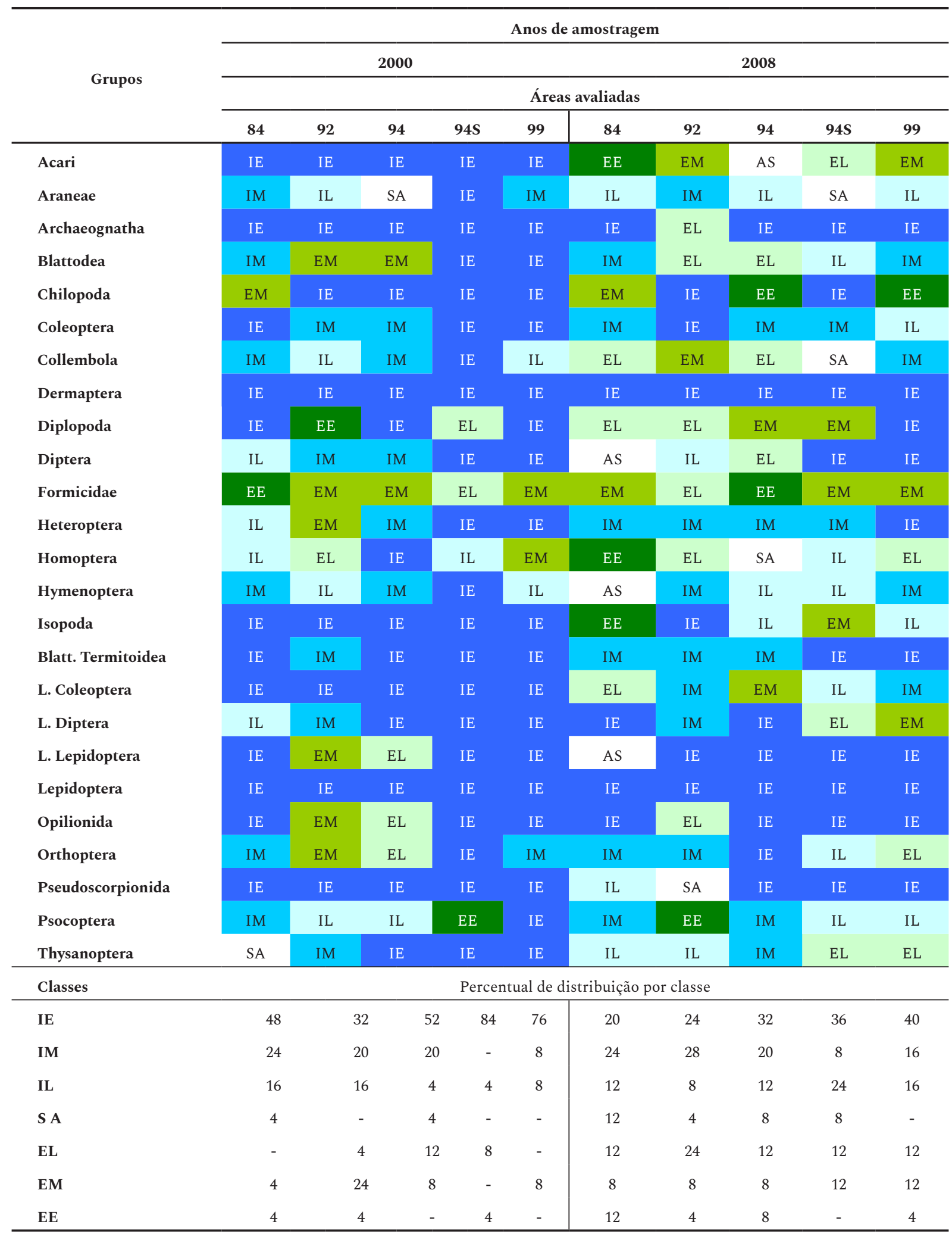

Fonte: Autores (2010)

Em que: IE = Inibição extrema; IM = Inibição moderada; IL = Inibição leve; AS = Sem alteração; EL = Estimulação leve; EM = Estimulação moderada; EE = Estimulação extrema. 
Figura 3 - Diagrama de ordenamento multivariado obtido com a técnica de NMS a partir dos grupos da fauna do solo amostrados em plantios sobre estéril de mineração de bauxita e mata nativa de 2000 a 2008, Porto Trombetas, PA

Figure 3 - Multivariate classification diagram obtained with the NMS technique from the soil fauna groups sampled in reforestations on bauxite mining subsoil and native forest from 2000 to 2008, Porto Trombetas, PA

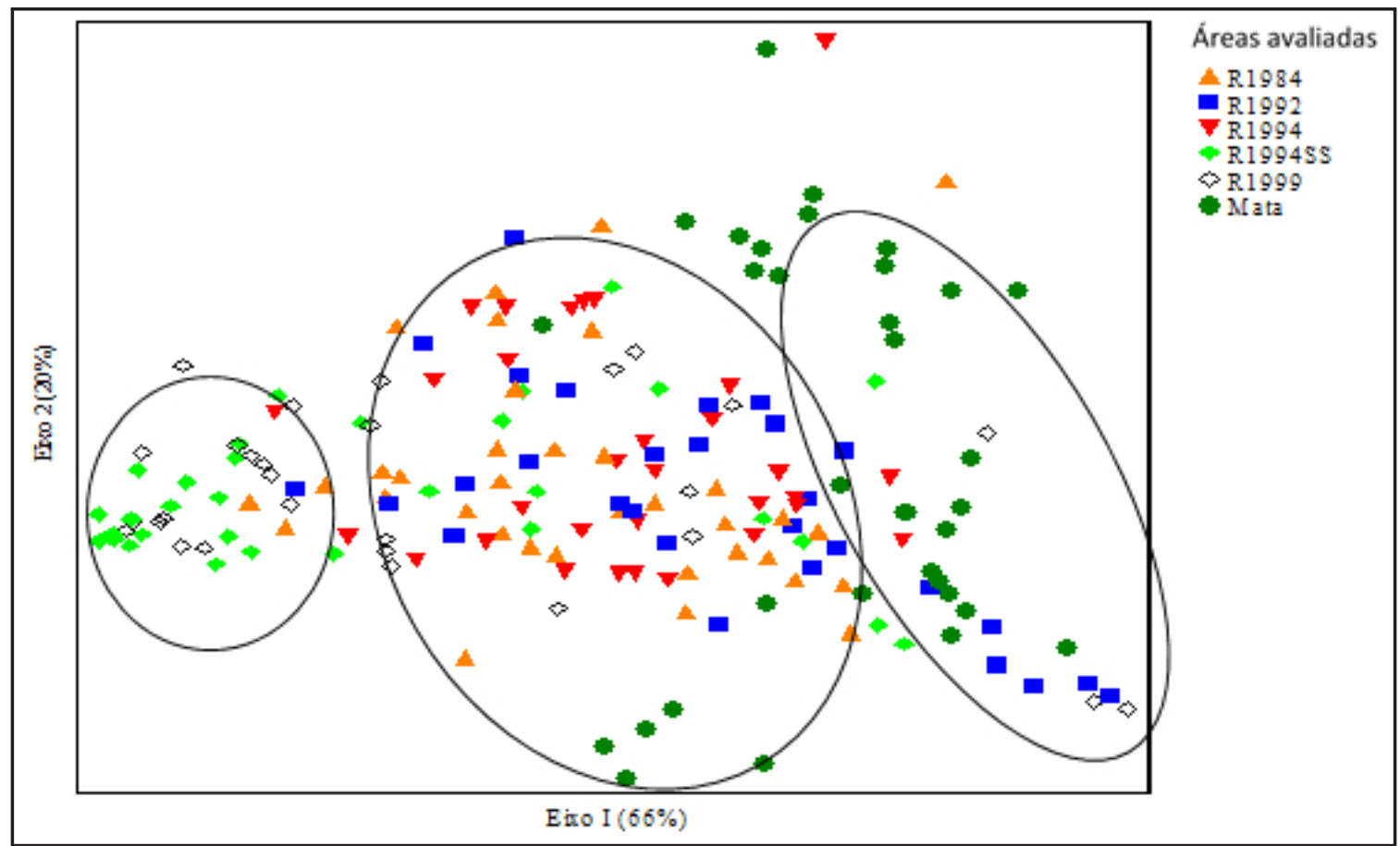

Fonte: Autores (2010)

\section{Conclusões}

O padrão de estruturação e diversidade das comunidades da fauna de solo apresenta grande variação ao longo dos anos. Nos plantios mais antigos, a comunidade de fauna edáfica está mais estruturada, enquanto nos mais recentes ou que não foi aplicado solo superficial, essa comunidade apresenta variações maiores e menor diversidade.

\section{Agradecimentos}

À UFRRJ pela oportunidade do curso de Pós-graduação, à Embrapa Agrobiologia pelas instalações e apoio no trabalho, ao ICMBio e a MRN pelas áreas trabalhadas e ao CNPq e Capes pela bolsa de estudos.

\section{Referências}

BAKKER, J. D. Increasing the utility of Indicator Species Analysis. Journal of Applied Ecology, London, v. 45, p. 1829-1835, 2008.

BIONDINI, M. E.; BONHAM, C. D.; REDENTE, E. F. Secondary successional patterns in a 
sagebrush (Artemisia tridentata) community as they relate to soil disturbance and soil biological activity. Vegetation, New York. v. 60, p. 25-36, 1985.

CHAER, G. M. et al. Nitrogen-fixing legume tree species for the reclamation of severely degraded lands in Brazil. Tree Physiology, Oxford, v. 31, n. 2, p. 139-149, 2011.

DORAN, J. W.; ZEISS, M. R. Soil health and sustainability: managing the biotic component of soil quality. Applied Soil Ecology, Netherlands, v. 15, p. 3-11, 2000.

DUFRENE, M.; LEGENDRE, P. Species assemblages and indicator species: the need for a flexible asymmetrical approach. Ecological Monographs, Washington, v. 67, p. 345-366, 1997.

FERRAZ, J. B. Soil factors influencing the reforestation on mining sites in Amazonia. In: LIETH, H.; LOHMAN, M. (ed.). Restoration of tropical forest ecosystems. Dordrecht: Kluwer Academic, 1993. p. 47-52.

GIÁCOMO, R. G. et al. Deposição de serapilheira e carbono em plantios de sabiá, andiroba e floresta secundária. Floresta, Paraná, v. 47, n. 2, p. 187-196, jul. 2017.

KRUSKAL, J. B. Multidimensional scaling by optimizing goodness of fit to a nonmetric hypotheses. Psychometrika, New York, v. 29, p. 1-27, 1964a.

KRUSKAL, J. B. Nonmetric multidimensional scaling: a numerical method. Psychometrika, New York, v. 29, p. 115-129, 1964b.

LAPA, R. P. A bauxita e o resíduo da bauxita. In: BOZELLI, R. L.; ESTEVES, F. A; ROLAND, F. Lago Batata: Impacto e recuperação de um ecossistema amazônico. Rio de Janeiro: IB-UFRJ; SBL, 2000. p. 25-36.

LIMA, K. D. R. de et al. Soil fauna as bioindicator of recovery of degraded areas in the caatinga biome. Revista Caatinga, Mossoró, v. 30, n. 2, p. 401-411, jun. 2017.

LIMA, T. M.; NEVES, C. A. R. (coord.). Sumário Mineral. Brasília: DNPM, 2016. 136 p.

MARTINS, E.M. et al. O uso de sistemas agroflorestais diversificados na restauração florestal na Mata Atlântica. Ciência Florestal, Santa Maria, v. 29, n. 2, p. 632-648, abr./jun. 2019.

MCGEOCH, M. A. The selection, testing and application of terrestrial insects as bioindicators. Biological Reviews, Cambridge, v. 73, p. 181-201, 1998.

MINERAÇÃO RIO DO NORTE. Perfil Litológico da Jazida de Porto Trombetas. [S. l.], 2008. Disponível em: http://www.mrn.com.br/index_1024.htm. Acesso em: 20 out. 2009.

MOLDENKE, A. R. Arthropods. In: WEAVER, R. W. et al. (ed.). Methods of Soil Analysis: Microbiological and Biochemical Properties. Madison: SSSA, 1994. pt. 2. 1121 p. (Soil Science Society of America Book Series, 5).

MOREIRA, J. F. Fauna do solo como bioindicador no processo de revegetação de áreas de mineração de bauxita em Porto Trombetas-PA.2010. Tese (Doutorado em Agronomia) Universidade Federal Rural do Rio de Janeiro, Seropédica, 2010.

SALOMÃO, R. P. Restauração florestal de precisão: dinâmica e espécies estruturantes. [S. l.]: Editora Novas Edições Acadêmicas, 2015. 404 p.

SALOMÃO, R. P.; ROSA, N. P.; MORAIS, K. A. C. Dinâmica de regeneração natural de árvores em áreas mineradas na Amazônia. Boletim do Museu Paraense Emílio Goeldi. Ciências Naturais, Belém, v. 2, n. 2, p. 85-139, 2007.

WARDLE, D. A. Impacts of disturbance on detritus food-webs in agroecosystems of contrasting tillage and weed management practices. In: BEGON, M.; FITTER, A. H. (ed.). Advances in ecological research. New York: Academic Press, 1995. v. 26. p. 105-185. 